ECOTOX - Brazil

\title{
Alterações do sistema de defesa antioxidativo em plântulas de Stizolobium aterrimum e Canavalia ensiformis expostas ao arsênio
}

\author{
K. J. T. Nascimento, ${ }^{1}$ J. A. Oliveira, ${ }^{1 *}$ J. Cambraia $^{1} \&$ M. A. Oliva ${ }^{2}$ \\ ${ }^{1}$ Departamento de Biologia Geral, Universidade Federal de Viçosa, Viçosa, 36570-000, Brazil \\ ${ }^{2}$ Departamento de Biologia Vegetal, Universidade Federal de Viçosa, Viçosa, 36570-000, Brazil
}

(Received November 24, 2008; Accepted July 07, 2010)

\begin{abstract}
RESUMO
O presente trabalho teve como objetivo avaliar as respostas antioxidantes em Stizolobium aterrimum e Canavalia ensiformis submetidas ao arsênio (As). Plântulas de C. ensiformis e $S$. aterrimum, com o primeiro par de folhas cotiledonares completamente expandidas, cultivadas em solução nutritiva de Clark, pH 5,5, foram tratadas com As nas concentrações de 0,0 e 1,5 mg. $\mathrm{L}^{-1}$, durante cinco dias. Após este período, determinou-se o efeito do As sobre a indução de estresse oxidativo e sobre parâmetros antioxidantes. A peroxidação de lipídios nas raízes e nas folhas sofreu acréscimos significativos, especialmente em S. aterrimum. Houve aumento na atividade das dismutases do superóxido (SODs), tanto nas raízes quanto nas folhas nas duas espécies. A atividade das peroxidases (PODs), nas folhas e raízes, reduziu em S. aterrimum, mas aumentou em C. ensiformis após exposição ao As. A atividade das redutases da glutationa (GRs) aumentou nas raízes das duas espécies, especialmente em S. aterrimum. Nas folhas, entretanto, a atividade das GRs aumentou em C. ensiformis, mas decresceu em S. aterrimum. Sob as condições experimentais da presente pesquisa, conclui-se que $C$. ensiformis apresenta mecanismos antioxidantes sobre os intermediários reativos de oxigênio (ROI) ligeiramente mais eficientes do que $S$. aterrimum.
\end{abstract}

Palavras-chave: arsênio, mecanismos antioxidantes, peroxidação de lipídios, intermediários reativos de oxigênio, mucunapreta, feijão-de-porco.

\section{ABSTRACT}

\section{Changes in antioxidative defense system of Stizolobium aterrimum and Canavalia ensiformis exposed to arsenic}

The present work evaluated the antioxidant responses of Stilozobium aterrimum and Canavalia ensiformis after exposure to arsenic (As). Plants of these two species with the first pair of fully expanded cotyledonary leaves, grown in nutrient solution, $\mathrm{pH} 5.5$, were treated with 0.0 and $1.5 \mathrm{mg} . \mathrm{L}^{-1}$ of As. After 5 days, the effects of As on lipid peroxidation and antioxidant enzymes were measured. Lipid peroxidation increased in both roots and leaves after As treatment, especially in S. aterrimum. Superoxide dismutase (SOD) activity increased in the two parts of the plants of both species with As treatment. Peroxidase (POX) activity, on the other hand, reduced with As treatment in S. aterrimum, but increased in C. ensiformis. Glutathione reductase (GR) increased in roots of both species, mainly in S. aterrimum. GR activity also increased in the leaves of $C$. ensiformis, but decreased in S. aterrimum. Under the experimental conditions of this study, C. ensiformis was considered to have antioxidant mechanisms slightly more efficient than $S$. aterrimum against reactive oxygen intermediates (ROIs).

Keywords: arsenic, antioxidative mechanisms, lipid peroxidation, reactive oxygen intermediates.

* Corresponding author: Juraci Alves de Oliveira; E-mail: jalves@ufv.br 


\section{INTRODUÇÃO}

O incremento de arsênio (As), metalóide com elevada toxicidade para a fauna e flora, nos ecossistemas terrestres e aquáticos, se deve a diversas ações antrópicas, tais como a atividade de mineração e o uso de pesticidas e fertilizantes na agricultura (Mandal \& Suzuki, 2002). Dentre as diversas tecnologias que visam recuperar os ambientes contaminados, a fitorremediação é uma das técnicas mais promissoras e, neste caso, envolve a utilização de espécies vegetais que melhor se adaptem a essas condições ambientais.

O As pode ocorrer em vários estados de oxidação ( -3 , 0 , +3 e +5), embora, sob condições aeróbias, a forma mais encontrada e disponível seja a pentavalente (arsenato) (Raquel \& Tena, 2005). O arsenato, quando absorvido, interfere nas reações de fosforilação, pois se liga ao ADP formando o ADP-As, provocando alterações no fluxo energético celular (Hugles, 2002). Dessa forma, o As contribui para o excesso de intermediários reativos de oxigênio (ROIs) que, também, podem ser produzidos durante a redução do arsenato a arsenito (Meharg \& Harttley-Whitaker, 2002; Mascher et al., 2002).

A indução de estresse oxidativo pelo As é um dos principais efeitos da toxicidade e injúria celular deste elemento em plantas, principalmente por causar a peroxidação de lipídios, (Sunkar et al., 2003; Raquel \& Tena, 2005). O dano celular se inicia com a peroxidação de ácidos graxos insaturados das membranas celulares (Singh et al., 2007), acarretando alteração de permeabilidade e flexibilidade e, ainda, a ruptura das estruturas celulares, conforme observado no sistema de membranas do cloroplasto (Li et al., 2006) e nas demais membranas celulares (Meharg, 1993).

O As promove alterações em diversos mecanismos bioquímicos dos vegetais, principalmente nos responsáveis pela tolerância das plantas ao estresse oxidativo. A ativação destes mecanismos é demonstrada pelo aumento na atividade de várias enzimas antioxidantes, destacando-se as dismutases do superóxido (SODs), as catalases (CATs), as peroxidases do ascorbato (APXs), as peroxidases totais (PODs) e as redutases da glutationa (GRs).

As SODs, removem os radicais superóxido $\left(\mathrm{O}_{2}^{\bullet-}\right)$, tendo o $\mathrm{H}_{2} \mathrm{O}_{2}$ como produto de reação (Gratão et al., 2005), sendo considerada a primeira na linha de defesa contra o estresse oxidativo. A remoção do $\mathrm{H}_{2} \mathrm{O}_{2}$ depende da atividade das PODs e GRs, dentre outras, consideradas fundamentais na manutenção da homeostase celular. A ação dessas enzimas evita a produção de radical hidroxil (HO•), gerado pelas reações de "Haber-Weiss" e "Fenton" (Asada, 1992).

Nas células das plantas, as PODs apresentam ampla distribuição e atuam na oxidação de vários compostos orgânicos e inorgânicos, na presença de $\mathrm{H}_{2} \mathrm{O}_{2}$ ou de hidroperóxidos orgânicos (Asada, 1992). As GRs participam do ciclo ascorbato-glutationa, sendo responsáveis pela manutenção dos níveis de glutationa na forma reduzida (Carlberg e Mannervik, 1985) e, portanto, consideradas cruciais para a manutenção do equilíbrio celular, uma vez que a regulação fina dos níveis de $\mathrm{H}_{2} \mathrm{O}_{2}$ é obtida por enzimas e metabólitos do ciclo do ascorbato-glutationa (Shigeoka et al., 2002).
Stizolobium aterrimum (mucuna-preta) Piper \& Tracy (1910) e Canavalia ensiformis (feijão-de-porco) (L.) DC. (1825) têm sido utilizadas em programas de fitorremediação, como fitoestabilizadoras de contaminantes (Procópio et al., 2005; Santos et al., 2007) e, apesar disso, existem poucas informações sobre o comportamento dessas espécies em áreas contaminadas com As. Portanto, o presente trabalho teve como objetivo avaliar as respostas antioxidativas em Stizolobium aterrimum e em Canavalia ensiformis expostas ao As.

\section{MATERIAL E MÉTODOS}

\section{Cultivo e aclimatação das plântulas}

Sementes de Stizolobium aterrimum e de Canavalia ensiformis, fornecidas pela Piraí Sementes, Piracicaba/SP, foram selecionadas para manter a homogeneidade de tamanho e forma, desinfetadas por 15 minutos em solução de hipoclorito de sódio 2,5\%, lavadas em água desmineralizada, e germinadas em cartuchos de papel "germiteste". Nas sementes de S. aterrimum foi feita a quebra de dormência com ácido sulfúrico, por 15 minutos, seguido de lavagem em água desmineralizada.

Após a germinação, quando as radículas atingiram comprimento de $3 \mathrm{~cm}, 40$ plântulas de cada espécie foram transferidas para recipientes de polietileno contendo $20 \mathrm{~L}$ de solução nutritiva de Clark (1975), pH 5,5, em sala de crescimento com temperatura e intensidade luminosa controladas $\left(25 \pm 2{ }^{\circ} \mathrm{C}\right.$, $\left.230 \mu \mathrm{E} . \mathrm{s}^{-1} \cdot \mathrm{m}^{-2}\right)$, sob fotoperíodo de 16 horas.

As plântulas das duas espécies, ao apresentarem o primeiro par de folhas cotiledonares expandidas, foram transferidas para vasos opacos de polietileno contendo $1,7 \mathrm{~L}$ de solução nutritiva de Clark (1975), pH 5,5, ajustado diariamente, e tratadas com As, na forma de $\mathrm{Na}_{2} \mathrm{HAsO}_{4} \cdot 7 \mathrm{H}_{2} \mathrm{O}$, nas concentrações de 0,0 e 1,5 mg.L. $\mathrm{L}^{-1}$, permanecendo por cinco dias. Transcorrido esse período, procedeu-se à coleta das folhas e raízes, as quais foram armazenadas a $-80^{\circ} \mathrm{C}$ até a execução das análises bioquímicas.

\section{Determinação da peroxidação de lipídios}

Amostras de 0,2 $\mathrm{g}$ de tecidos vegetais foram trituradas em nitrogênio líquido e homogeneizadas em $2 \mathrm{~mL}$ de ácido tricloroacético (TCA) $0,1 \%(\mathrm{p} / \mathrm{v})$, seguido de filtração através de quatro camadas de gaze, e centrifugação a 12.000 $x \mathrm{~g}$ por $15 \mathrm{~min}$. Todas as etapas necessárias ao processo de extração foram conduzidas a $4^{\circ} \mathrm{C}$. Alíquotas de $0,5 \mathrm{~mL}$ dos sobrenadantes foram adicionadas a $1,5 \mathrm{~mL}$ de uma solução de ácido tiobarbitúrico (TBA) 0,5\% (p/v) em TCA 20\% (p/v) e incubadas em banho-maria, a $95^{\circ} \mathrm{C}$. Após $2 \mathrm{~h}$, a reação foi paralisada com a transferência dos tubos de ensaio para banho de gelo. Posteriormente, foram centrifugadas a $9.000 \mathrm{xg}$ por 10 min e a absorvância dos sobrenadantes determinadas a $532 \mathrm{~nm}$. A absorvância inespecífica a $600 \mathrm{~nm}$ foi medida e subtraída dos resultados obtidos das amostras. A concentração do complexo aldeído malônico-TBA (TBARs) foi calculada usando o coeficiente de extinção molar de $155 \mathrm{mM}^{-1} \mathrm{~cm}^{-1}$ (Heath \& Packer, 1968). 


\section{Determinação das atividades das enzimas dismutases do superóxido (SODs; EC 1.15.1.1), peroxidases (PODs; EC 1.11.7) e redutases da glutationa (GRs; EC 1.6.4.2)}

Amostras de 0,3 g de massa fresca de raízes e folhas foram maceradas em nitrogênio líquido, seguido da adição de $2 \mathrm{~mL}$ de meio de homogeneização, filtração através de quatro camadas de gaze e centrifugação a $12.000 \mathrm{xg}$, por 15 minutos, a $4^{\circ} \mathrm{C}$. Os sobrenadantes constituíram os extratos enzimáticos brutos utilizados na determinação das atividades das enzimas. O meio de homogeneização foi constituído de tampão fosfato de potássio $0,1 \mathrm{M}, \mathrm{pH} 6,8$, ácido etilenodiaminotetracético (EDTA) 0,1 mM, fluoreto de fenilmetilsulfônico (PMSF) $1 \mathrm{mM}$ e polivinilpolipirrolidona (PVPP) $1 \%(\mathrm{p} / \mathrm{v})$ para as enzimas SODs e POXs (Peixoto et al., 1999). Para as GRs, o meio de homogeneização foi constituído de tampão fosfato de potássio 0,1 M, pH 7,5, EDTA $1 \mathrm{mM}$, dithiothreitol $2 \mathrm{mM}$ (DTT), PMSF $1 \mathrm{mM}$ e PVPP 1\% (p/v) (Carlberg \& Mannervik, 1985).

A atividade das SODs foi determinada pela adição de $30 \mu \mathrm{L}$ do extrato enzimático bruto a $2,97 \mathrm{~mL}$ de meio de reação, constituído de tampão fosfato de sódio $50 \mathrm{mM}, \mathrm{pH} 7,8$, contendo metionina $13 \mathrm{mM}$, azul de p-nitro tetrazólio (NBT) $75 \mu \mathrm{M}$, EDTA $0,1 \mathrm{mM}$ e riboflavina $2 \mu \mathrm{M}$ (Del Longo et al., 1993). A reação foi conduzida a $25^{\circ} \mathrm{C}$, sob iluminação de lâmpada de $15 \mathrm{~W}$. Após 5 min de exposição à luz, a iluminação foi interrompida e a formazana azul, produzida pela fotorredução do NBT, medida a $560 \mathrm{~nm}$ (Giannopolitis \& Ries, 1977). A absorvância a $560 \mathrm{~nm}$ de meio de reação exatamente igual ao anterior, mas mantido no escuro por igual tempo, serviu de branco e foi subtraído da leitura da amostra que recebeu iluminação. Uma unidade de SOD foi definida como a quantidade de enzima necessária para inibir em $50 \%$ a fotorredução do NBT (Peixoto et al., 1999).

A atividade das PODs foi determinada pela adição de 100 $\mu \mathrm{L}$ do extrato enzimático a $2,9 \mathrm{~mL}$ de meio de reação constituído de tampão fosfato de potássio $25 \mathrm{mM}, \mathrm{pH} \mathrm{6,8,} \mathrm{pirogalol} 20$ $\mathrm{mM}$ e $\mathrm{H}_{2} \mathrm{O}_{2} 20 \mathrm{mM}$. O acréscimo na absorvância a $420 \mathrm{~nm}$, à temperatura de $25^{\circ} \mathrm{C}$, foi medido durante o primeiro minuto de reação, pela produção de purpurogalina. A atividade das PODs foi calculada utilizando o coeficiente de extinção molar de 2,47 $\mathrm{mM}^{-1} . \mathrm{cm}^{-1}$ (Chance \& Maehley, 1955).

A atividade das GRs foi determinada pela adição de 100 $\mu \mathrm{L}$ de extrato enzimático bruto a $0,9 \mathrm{~mL}$ de um meio de reação constituído de tampão fosfato de potássio $0,1 \mathrm{M}, \mathrm{pH}$ 7,5, EDTA $1 \mathrm{mM}$, glutationa oxidada (GSSG) $1 \mathrm{mM}$ e NADPH $0,1 \mathrm{mM}$ (em tampão TRIS-HCl 0,5 mM, pH 7,5), segundo Carlberg \& Mannervik (1985). O decréscimo na absorvância a 340 $\mathrm{nm}$, à temperatura de $30^{\circ} \mathrm{C}$, foi medido no primeiro minuto após o início da reação. A atividade enzimática foi calculada utilizando-se o coeficiente de extinção molar de $6,22 \mathrm{mM}^{-1}$. $\mathrm{cm}^{-1}$ (Foyer \& Halliwell, 1976).

\section{Delineamento Estatístico}

$\mathrm{O}$ experimento foi realizado em blocos ao acaso em esquema fatorial $2 \times 2$ ( 2 espécies $\times 2$ concentrações de As), com três repetições. Os dados foram submetidos à ANOVA, utilizando o programa estatístico "SAEG", da Fundação Arthur Bernardes, da Universidade Federal de Viçosa, versão 9.0.

\section{RESULTADOS E DISCUSSÃO}

As plantas das duas espécies, submetidas ao tratamento com As, sofreram acréscimos significativos nos teores de malondialdeído (TBARs) nos órgãos analisados (Figura 1a). Em Stizolobium aterrimum houve incrementos de $59 \%$ e de $81 \%$, nas folhas e nas raízes, respectivamente. Em Canavalia ensiformis observou-se incremento de $47 \%$ nas folhas e de $30 \%$ nas raízes. Estes efeitos tóxicos do As foram observados, também, em outras espécies, como Avena sativa (Stoeva \& Bineva, 2003), Phaseolus vulgaris (Stoeva et al., 2005), Pteris sp (Singh et al., 2006) e em Phaseolus aureus (Singh et al., 2007).

$\mathrm{O}$ aumento nos teores de malondialdeído, devido à peroxidação de ácidos graxos poliinsaturados (Montillet et al., 2005), evidencia a ocorrência de danos às membranas celulares, as quais são consideradas os principais locais de ação de elementos tóxicos, incluindo o As (Meharg, 1993). O As induz o aumento na produção de ROIs (Cakmak \& Horst, 1991), que promovem a peroxidação de lipídios, resultando em desorganização da estrutura das membranas (Stoeva \& Bineva, 2003).

O estresse oxidativo induzido pelo As pode ser regulado pela atividade de várias enzimas do sistema antioxidativo (Sunkar et al., 2003; Raquel \& Tena, 2005), como as dismutases do superóxido, as peroxidases e as redutases da glutationa, as quais mantém baixos os níveis de ROIs, colaborando para a manutenção da homeostase celular.

A exposição das plantas ao As resultou em aumento na atividade das SODs, tanto nas folhas quanto nas raízes de ambas as espécies (Figura 1B). Em S. aterrimum observou-se aumento de $6 \%$ e $19 \%$, nas folhas e nas raízes, respectivamente, e, em C. ensiformis, esse aumento foi de $15 \%$ nas folhas e de $21 \%$ nas raízes. Semelhante ao observado neste trabalho, o aumento na atividade das SODs tem sido relatado para diversas espécies vegetais expostas ao As, como em Zea mays (Mylona et al, 1998), Holcus lanatus (Hartley-Whitaker et al., 2001), Trifolium pratense (Mascher et al., 2002), Pteris vittata (Cao \& Ma, 2004) e Phaseolus aureus (Singh et al., 2007).

O maior incremento na atividade dessa enzima, especialmente em folhas de $C$. ensiformis, evidencia a produção de $\mathrm{O} \overline{2}$ resultante da ação tóxica do As nas plantas. A atividade mais expressiva da SOD em C. ensiformis proporcionou menores teores de malondialdeído, especialmente nas folhas. Observa-se, porém, que a ação dessa enzima não foi suficiente para evitar os danos oxidativos causados pelo As em ambas as espécies. Portanto, a atuação isolada das SODs não é suficiente para manutenção da homeostase celular frente à peroxidação de lipídios, uma vez que a ação dessa enzima resulta na produção de $\mathrm{H}_{2} \mathrm{O}_{2}$, que também é tóxico e, portanto, necessita da participação conjunta de outras enzimas capazes de degradar $\mathrm{o}_{2} \mathrm{O}_{2}$, como as peroxidases (Apel \& Hirt, 2004).

Nas folhas e raízes de $S$. aterrimum expostas ao As verificou-se decréscimos de 24 e de $27 \%$, respectivamente, na atividade de PODs. Em C. ensiformis, por sua vez, houve significativos incrementos da atividade das PODs, sendo de $137 \%$ nas folhas e 11\% nas raízes (Figura 1C).

A redução na atividade das PODs observada em $S$. aterrimum indica que o estresse oxidativo ultrapassou a 

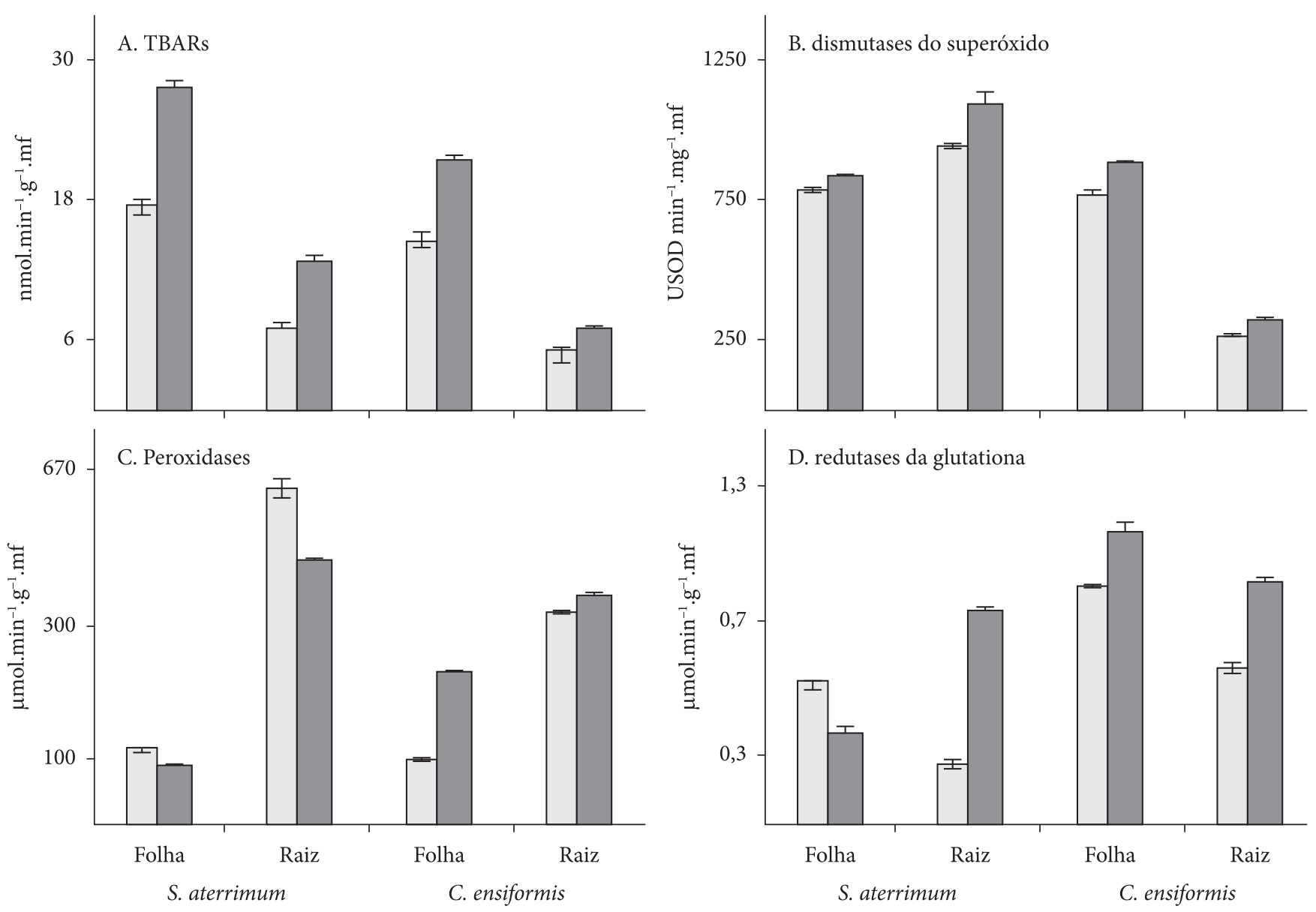

Figura 1 - Produção média ( \pm erro padrão) de TBARs (A), atividade das enzimas dismutases do superóxido (SODs), peroxidases (PODs) e redutases da glutationa (GRs) (B, C e D, respectivamente), nas folhas e nas raízes de S. aterrimum e C. ensiformis, após cinco dias de exposição ao As (- - 0,0 e $\square-1,5 \mathrm{mg} \mathrm{L}^{-1}$ ), em solução nutritiva. As barras representam o erro padrão da média.

capacidade de tolerância das plantas, resultando em inibição enzimática. A inativação dessa enzima pode se dar pela ligação do As aos seus grupos tióis (Meharg \& Hartley-Whitaker, 2002) ou via oxidação (Dietz, 2003). Dessa forma, admite-se que outros mecanismos enzimáticos e não-enzimáticos precisam ser ativados para remoção do excesso de $\mathrm{H}_{2} \mathrm{O}_{2}$ nesta espécie (Cao \& Ma 2004).

O aumento na atividade de PODs, conforme observado em plantas de C. ensiformis expostas ao As, tem sido relatado para outras espécies, como em Trifolium pratense (Mascher et al., 2002), Pteris vittata (Cao \& Ma, 2004), Solanum lycopersicum (Miteva et al., 2005) e Phaseolus vulgaris (Stoeva et al., 2005) e indica ser essa enzima importante componente do mecanismo de proteção metabólica contra o excesso de $\mathrm{H}_{2} \mathrm{O}_{2}$ em C. ensiformis.

Em S. aterrimum observou-se redução de $39 \%$ na atividade das GRs nas folhas e acréscimo de $257 \%$ nas raízes. Em C. ensiformis a atividade de GRs aumentou tanto em folhas como em raízes, sendo de 23 e de $54 \%$, respectivamente (Figura 1d). As respostas enzimáticas de plantas expostas ao As podem variar consideravelmente, podendo ocorrer aumento, conforme observado em Brassica juncea (Khan et al., 2008), ou redução, semelhante à observada em folhas de Ceratophyllum demersum (Mishra et al., 2008).

O As pode ter contribuído para a formação de níveis elevados de conjugados glutationa-As nas folhas de S. aterrimum, os quais possuem forte ação inibitória sobre as GRs (Foyer et al., 1997). Outras hipóteses para explicar a redução na atividade das GRs em $S$. aterrimum seriam a inativação desta enzima via oxidação ou pela ligação do As aos seus grupos SH (Meharg \& Hartley-Whitaker, 2002; Dietz, 2003).

As GRs apresentam importante função na proteção contra o estresse oxidativo, pois participam do ciclo ascorbato-glutationa sustentando a alta taxa de glutationa reduzida em relação à glutationa oxidada (Mittler, 2002). Assim, o incremento da atividade das GRs indica participação efetiva desta enzima no metabolismo antioxidativo das duas espécies, especialmente em C. ensiformis, sendo imprescindível para a manutenção da homeostase celular.

\section{CONCLUSÕES}

A peroxidação de lipídios aumentou nas raízes e nas folhas das duas espécies expostas ao As, sendo que Stizolobium aterrimum apresentou maior peroxidação de lipídios, tanto 
em raízes quanto em folhas. Assim, de modo geral, com base na peroxidação de lipídios e nas atividades das enzimas antioxidativas, tanto nas raízes como nas folhas, conclui-se que Canavalia ensiformis apresenta mecanismo de defesa enzimático sobre os ROIs ligeiramente mais eficiente do que S. aterrimum.

Agradecimentos: Os autores agradecem a CAPES e FAPEMIG pelo apoio financeiro.

\section{REFERÊNCIAS BIBLIOGRÁFICAS}

APEL, K. \& HIRT H., 2004, Reactive oxygen species: metabolism, oxidative stress, and signal transduction. Plant Biol., 55: 373399. doi: 10.1146/annurev.arplant.55.031903.

ASADA, K., 1992, Ascorbate peroxidase: a hydrogen peroxidescavenging enzyme in plants. Physiol. Plant., 85: 235-241.

CAKMAK, W. \& HORST, J., 1991, Effects of aluminum on lipid peroxidation, superoxide dismutase, catalase and peroxidase activities in root tips of soybean (Glycine max). Physiol. Plant., 83: 463-772.

CAO, X. \& MA, L.Q., 2004, Antioxidative responses to arsenic in the arsenic-hyperaccumulator Chinese brake fern (Pteris vittata L.). Environ. Pollut., 128: 317-325. doi:10.1016/j. envpol.2003.09.018

CARLBERG, I. \& MANNERVIK, B., 1985, Glutathione reductase. Method. Enzymol., 113: 484-495. doi:10.1016/S00766879(85)13062-4

CHANCE, B. \& MAEHLEY, A.C., 1955, Assay of catalase and peroxidases. Method. Enzymol., 11: 764-775. doi:10.1016/ S0076-6879(55)02300-8

CLARK, R.B., 1975, Characterization of phosphatase of intact maize roots. J. Agric. Food Chem., 23: 458-460. doi:10.1021/ jf60199a002

DEL LONGO, O.T., GONZÁLEZ, A., PASTORI, G.M., \& TRIPPI, V.S., 1993, Antioxidant defenses under hyperoxygenic and hyperosmotic conditions in leaves of two lines of maize with differential sensitivity to drought. Plant Cell Physiol., 34: 10231028.

DIETZ, R.L.M., 2003, Plant peroxiredoxins. Plant Biol., 54: 93-107.

FOYER, C.H. \& HALLIWELL, B., 1976, The presence of glutathione and glutathione reductase in chloroplasts: a proposed role in ascorbic acid metabolism. Planta., 133: 21-25. doi: 10.1007/BF00386001

FOYER, C.H., LOPEZ-DELGADO, H., DATE, J.F. \& SCOTT, I.M., 1997, Hydrogen peroxide and glutathione associated mechanisms of acclimatory stress tolerance and signaling. Physiol. Plant., 100: 241-254.

GIANNOPOLITIS, C.N. \& RIES, S.K., 1977, Superoxide dismutases. I. Occurrence in higher plants. Plant Physiol., 59: 309-314. 10.1104/pp.59.2.309

GRATÃO, P.L., POLLE, A., LEA, P.J. \& AZEVEDO, A.R., 2005, Making the life of heavy metal-stressed plants a little easier. Funct. Plant Biol., 32: 481-494. doi: 10.1071/FP05016

HARTLEY-WHITAKER, J., AINSWORT, G. \& MEHARG, A.A., 2001, Copper and arsenate-induced oxidative stress in Holcus lanatus L. clones with differential sensitivity. Plant Cell Environ., 24: 713-722. doi: 10.1046/j.0016-8025.2001.00721.x

HEATH, R.L. \& PACKER, L., 1968, Photoperoxidation in isolated chloroplast. I. Kinetics and stoichiometry of fatty acid peroxidation. Arch. Biochem. Biophys., 125: 189-198. doi: doi:10.1016/0003-9861(68)90654-1

HUGHES, M.F., 2002, Arsenic toxicity and potential mechanisms of action. Toxicol. Lett., 33: 1-16. doi:10.1016/S03784274(02)00084-X

KHAN I, AHMAD A, IQBAL, M., 2009, Modulation of antioxidant defense system of arsenic detoxification in Indian mustards. Ecotoxicol. Environ. Saf., 72: 626-634. doi:10.1016/j. ecoenv.2007.11.016

LI, W-X., Chen, T-B., Huang, Z-C., Lei, M. \& Liao X-Y., 2006, Effect of arsenic on chloroplast ultrastructure and calcium distribution in arsenic hyperaccumulator Pteris vittata L. Chemosphere., 62: 803-809. doi:10.1016/j.chemosphere.2005.04.055

MANDAL, B.K. \& SUZUKI, K.T., 2002, Arsenic round the world: a review. Talanta, 58: 201-235. doi:10.1016/S00399140(02)00268-0

MASCHER, R., LIPPMANN, B., HOLZINGER, S. \& BERGMANN, H., 2002, Arsenate toxicity: effects on oxidative stress response molecules and enzymes in red clover plants. Plant Sci., 163: 961969. doi:10.1016/S0168-9452(02)00245-5

MEHARG, A.A., 1993, The role of plasmalemma in metal tolerance in angiosperms: Minireview. Physiol. Plantarum, 88: 91-198. doi: 10.1111/j.1399-3054.1993.tb01777.x

MEHARG, A.A. \& HARTLEY-WHITAKER, J., 2002, Arsenic uptake and metabolism in arsenic resistant and nonresistant plant species. New Phytol., 154: 29-43. doi: 10.1046/j.14698137.2002.00363.x

MISHRA, S., SRIVASTAVA, S., TRIPATHI, R.D. \& TRIVEDI, P.K., 2008, Thiol metabolism and antioxidant systems complement each other during arsenate detoxification in Ceratophyllum demersum L. Aquat. Toxicol., 86: 205-215. doi:10.1016/j. aquatox.2007.11.001

MITTLER, R., 2002, Oxidative stress, antioxidants and stress tolerance. Plant Sci., 7: 405-410.

MiteVA, E., HRISTOVA, D., NENOVA, V. \& MANEVA, S., 2005, Arsenic as a factor affecting virus infection in tomato plants: changes in plant growth, peroxidase activity and chloroplast pigments. Sci. Hortic., 105: 343-358. doi:10.1016/j. scienta.2005.01.026

MONTILLET, J-L., CHAMNOGPOL, S., RUSTÉRUCCI, C., DAT, J., VAN, DE COTTE, B., AGNEL, J-P., BATTESTI, C., INZÉ, D., VAN, B.F. \& TRIANTAPHYLIDES, C., 2005, Fatty acid hypersensitive and $\mathrm{H}_{2} \mathrm{O}_{2}$ in the execution of hypersensitive cell death in tobacco leaves. Plant Physiol., 138: 1516-1526. doi:10.1104/pp.105.059907

MYLONA, P.V., POLIDOROS, A.N. \& SCANDALIOS, J.G., 1998, Modulation of antioxidant responses by arsenic in maize. Free Radical Biol. Med., 25: 576-585. doi:10.1016/S08915849(98)00090-2

PEIXOTO, P.H.P., CAMBRAIA, J., SANT'ANA, R., MOSQUIM, P.R. \& MOREIRA, M.A., 1999, Aluminum effects on lipid peroxidation and on the activities of enzymes of oxidative metabolism in sorghum. Rev. Bras. Fisiol. Veg., 11: 137-143.

PROCÓPIO, S.O., SANTOS, J.B., SILVA, A.A., PIRES, F.R., RIBEIRO, J.J.I. \& SANTOS, E.A., 2005, Potential of plant species for remediation of trifloxysulfuron-sodium. Planta Daninha, 23: 9-16. doi: 10.1590/S0100-83582005000100002

RAQUEL, R. \& TENA, M., 2005, Proteome analysis of maize roots reveals that oxidative stress is a main contributing factor to plant arsenic toxicity. Phytochemistry, 66: 1519-1528. doi:10.1016/j. phytochem.2005.05.003 
SANTOS, E.A., SANTOS, J.B., FERREIRA, L.R., COSTA, M.D. \& SILVA, A.A., 2007, Phyto-stimulation by Stizolobium aterrimum as remediation of soil contaminated with trifloxysulfuronsodium. Planta Daninha, 25: 259-265.

SHIGEOKA, S., ISHIKAWA, T., TAMOI, M., MIYAGAWA, Y., TAKEDA, T., YABUTA, Y. \& YOSHIMURA, K., 2002, Regulation and function of ascorbato peroxidase isoenzymes. $J$. Exp. Bot., 53: 1305-1319. doi:10.1093/jexbot/53.372.1305

SINGH, H.P., BATISH, D.R., KOHLI, R.K. \& ARORA, K., 2007, Arsenic-induced root growth inhibition in mung bean (Phaseolus aureus Roxb.) is due to oxidative stress resulting from enhanced lipid peroxidation. Plant Growth Regul., 53: 65-73. doi: 10.1007/ s10725-007-9205-z
SINGH,N., MA,L.Q., SRIVASTAVA, M.\& RATHINASABAPATHI, B., 2006, Metabolic adaptations to arsenic-induced oxidative stress in Pteris vittata L and Pteris ensiformis L. Plant Sci., 170: 274-282. doi:10.1016/j.plantsci.2005.08.013

STOEVA, N., BEROVA, M. \& ZLATEV, Z., 2005, Effect of arsenic on some physiological parameters in bean plants. Biol. Plantarum., 49: 293-296. doi: 10.1007/s10535-005-3296-z

STOEVA, N. \& BINEVA, T., 2003, Oxidative changes and photosynthesis in oat plants grown in As-contaminated soil. Bulg. J. Plant Physiol., 29: 87-95.

SUNKAR, R., BARTELS, D. \& KIRCH, H-H., 2003, Overexpression of a stress-inducible aldehyde dehydrogenase gene from Arabidopsis thaliana in transgenic plants improves stress tolerance. Plant J., 35: 452-464. 